\title{
RR LYRAE VARIABLES IN TWO FIELDS IN THE SPHEROID OF M31*
}

\author{
Ata Sarajedini ${ }^{1}$, Conor L. Mancone ${ }^{1}$, Tod R. Lauer ${ }^{2}$, Alan Dressler $^{3}$, Wendy Freedman $^{3}$, S. C. Trager ${ }^{4}$, \\ Carl Grillmair ${ }^{5}$, and Kenneth J. Mighell ${ }^{2}$ \\ ${ }^{1}$ Department of Astronomy, University of Florida, Gainesville, FL 32611, USA; ata@ astro.ufl.edu, cmancone@astro.ufl.edu \\ ${ }^{2}$ National Optical Astronomy Observatory, P.O. Box 26732, Tucson, AZ 85726, USA; tlauer@noao.edu, kmighell@noao.edu \\ ${ }^{3}$ Observatories of the Carnegie Institution of Washington, Pasadena, CA 91101, USA; dressler@ociw.edu, wendy@ociw.edu \\ ${ }^{4}$ Kapteyn Astronomical Institute, University of Groningen, NL-9700 AV Groningen, Netherlands; sctrager@astro.rug.nl \\ 5 Spitzer Science Center, Pasadena, CA 91125, USA; carl@ipac.caltech.edu \\ Received 2008 October 21; accepted 2009 April 25; published 2009 June 1
}

\begin{abstract}
We present Hubble Space Telescope observations taken with the Advanced Camera for Surveys Wide Field Channel of two fields near M32-between 4 and $6 \mathrm{kpc}$ from the center of M31. The data cover a time baseline sufficient for the identification and characterization of 681 RR Lyrae variables of which 555 are ab-type and 126 are c-type. The mean magnitude of these stars is $\langle V\rangle=25.29 \pm 0.05$, where the uncertainty combines both the random and systematic errors. The location of the stars in the Bailey diagram and the ratio of c-type RR Lyraes to all types are both closer to RR Lyraes in Oosterhoff type I globular clusters in the Milky Way as compared with Oosterhoff II clusters. The mean periods of the ab-type and c-type RR Lyraes are $\left\langle P_{\mathrm{ab}}\right\rangle=0.557 \pm 0.003$ and $\left\langle P_{\mathrm{c}}\right\rangle=0.327 \pm 0.003$, respectively, where the uncertainties in each case represent the standard error of the mean. When the periods and amplitudes of the ab-type RR Lyraes in our sample are interpreted in terms of metallicity, we find the metallicity distribution function to be indistinguishable from a Gaussian with a peak at $\langle[\mathrm{Fe} / \mathrm{H}]\rangle=-1.50 \pm 0.02$, where the quoted uncertainty is the standard error of the mean. Using a relation between RR Lyrae luminosity and metallicity along with a reddening of $E(B-V)=0.08 \pm 0.03$, we find a distance modulus of $(m-M)_{0}=24.46 \pm 0.11$ for M31. We examine the radial metallicity gradient in the environs of M31 using published values for the bulge and halo of M31 as well as the abundances of its dwarf spheroidal companions and globular clusters. In this context, we conclude that the RR Lyraes in our two fields are more likely to be halo objects rather than associated with the bulge or disk of M31, in spite of the fact that they are located at 4-6 kpc in projected distance from the center.
\end{abstract}

Key words: galaxies: individual (M31) - galaxies: spiral - galaxies: stellar content - stars: variables: other

Online-only material: color figures, figure set, machine-readable and VO tables

\section{INTRODUCTION}

Pulsating variable stars such as RR Lyraes are powerful probes useful for investigating the properties of stellar populations. The mere presence of RR Lyraes among a population of stars suggests an ancient origin since ages older than $\sim 10$ Gyr are required to produce RR Lyrae variables. Their periods and amplitudes are a reflection of the metal abundance of the population. Along with their incredible usefulness, RR Lyraes are also relatively straightforward to identify and characterize. This is because of their short periods and the distinct light curve shapes of the ab-types, which pulsate in the fundamental mode and exhibit a relatively rapid rise to maximum and a gradual decline to minimum. This is in contrast to the c-type RR Lyraes which pulsate in the first harmonic and show light curves that are more akin to sine curves. In spite of the great potential RR Lyraes hold as astrophysical tools, they have not been widely studied in our nearest large neighbor galaxy, Andromeda.

One of the first studies attempting to identify RR Lyraes in M31 was that of Pritchet \& van den Bergh (1987). They used the Canada-France-Hawaii $3.6 \mathrm{~m}$ telescope to observe a field at a distance of $9 \mathrm{kpc}$ from the center of M31 along the minor axis partially overlapping the field observed by Mould \& Kristian (1986). They identified 30 RR Lyrae candidates and were able to estimate periods for 28 of them. These ab-type variables have

\footnotetext{
* Based on observations taken with the NASA/ESA Hubble Space Telescope, obtained at the Space Telescope Science Telescope.
}

a mean period of $\left\langle P_{\mathrm{ab}}\right\rangle=0.548$ days. The photometric errors in their data prevented them from identifying the lower amplitude c-type RR Lyraes.

The RR Lyrae variables in M31 globular clusters have been investigated by Clementini et al. (2001, cf., Contreras et al. 2008). They used the Wide Field Planetary Camera 2 (WFPC2) onboard the Hubble Space Telescope (HST) to make the first tentative detection of RR Lyraes in G11, G33, G64, and G322, finding two, four, 11, and eight variables, respectively. Detection and characterization of these stars in globular clusters are more challenging than in the M31 field because of the increased crowding.

Returning to the work on field RR Lyraes, Dolphin et al. (2004) observed the same field as Pritchet \& van den Bergh (1987) using the WIYN $3.5 \mathrm{~m}$ on Kitt Peak. They found 24 RR Lyrae stars with a completeness fraction of $24 \%$, suggesting that their $\sim 100 \operatorname{arcmin}^{2}$ field could contain $\sim 100$ RR Lyraes resulting in a density of one RR Lyrae per square arcmin. This is much less than the value of $\sim 17 \mathrm{arcmin}^{-2}$ found by Pritchet \& van den Bergh (1987). They also noted for the first time that the mean metallicity of the M31 RR Lyraes seemed to be significantly lower than that of the M31 halo. The work of Durrell et al. (2001) had reported a peak value of $[M / H] \sim-0.8$ for the M31 halo. Dolphin et al. (2004) were not able to reconcile this abundance value with the distance implied by the mean magnitude of their RR Lyrae sample.

The first definitive work on the RR Lyraes of M31 was published by Brown et al. (2004, hereafter B2004) and made use of $\sim 84 \mathrm{hr}$ of imaging time (250 exposures over 41 days) 
Table 1

Observing Log

\begin{tabular}{cccccccc}
\hline \hline Field & R.A. (J2000) & Decl. (J2000) & Starting Date & Data Sets & Filter & Exp Time & HJD Range (+2 453 000) \\
\hline 1 & 004241.2 & 404638 & 2005 Sep 22 & J9H905, J9H906, & F606W & $16 \times 1136 \mathrm{~s}$, & 635.97 to 638.25 \\
& & & & J9H907, J9H908 & & $16 \times 1177 \mathrm{~s}$ & \\
2 & 004320.8 & 405725 & 2006 Feb 9 & J9H913, J9H914, & F606W & $16 \times 1136 \mathrm{~s}$, & 775.88 to 778.96 \\
& & & J9H915, J9H916 & & $16 \times 1177 \mathrm{~s}$ & \\
\hline
\end{tabular}

with the Advanced Camera for Surveys onboard HST. Their field was located along the minor axis of M31 approximately 11 $\mathrm{kpc}$ from its center. Their analysis revealed a complete sample of RR Lyrae stars consisting of 29 ab-type variables and 25 c-type. The periods of these stars suggest a mean metallicity of $[\mathrm{Fe} / \mathrm{H}] \sim-1.6$ for the old population in the Andromeda halo. This is qualitatively consistent with the assertions of Dolphin et al. (2004) regarding the metal abundance of the M31 halothat it is lower than the value suggested by the work of Durrell et al. (2001). More recent work has shown that the M31 halo extends from 30 to $165 \mathrm{kpc}$ (Guhathakurta et al. 2005; Irwin et al. 2005) and has a metallicity that is actually closer to that of the Milky Way halo (Kalirai et al. 2006; Koch et al. 2008).

There is one more paper of note related to this topic and that is the work of Alonso-García et al. (2004). They used the WFPC2 onboard HST to image a field $\sim 3.5$ arcmin to the east of M32 and compared it with a control field that samples the M31 field stars well away from M32. They identify variable stars that they claim are RR Lyraes belonging to M32 therefore suggesting that M32 possesses a population that is older than $\sim 10$ Gyr. They were not able to classify the RR Lyraes or derive periods and amplitudes for them so their results are not directly comparable to ours.

This review of the literature reveals a significant deficiency in the spatial coverage of RR Lyrae studies in the vicinity of M31. Given the great astrophysical utility of RR Lyrae variables and the expansive size of M31 on the sky, it is clear that a survey of these stars sampling a diversity of regions in Andromeda will provide valuable insights into the star formation and chemical enrichment history of our nearest spiral neighbor.

With this in mind, this paper presents the results of archival HST Advanced Camera for Surveys (HST/ACS) observations showcasing the RR Lyrae population in the inner regions of the M31 spheroid. The following section describes the observational material and the photometric procedure. We move on to describe the technique used to identify and characterize the variable stars in Section 3. The results of this study are described in Section 4, and a discussion of these results within the broader context of the M31 halo is presented in Section 5. Our conclusions are then summarized in Section 6 .

\section{OBSERVATIONS}

The observations used in the present study were obtained with the $H S T /$ ACS in parallel with the imagery conducted by program GO-10572. The primary goal of this program was to obtain a deep color-magnitude diagram of the envelope of M32, using the high-resolution channel (HRC) of the ACS. The total envelope exposure was 32 orbits, split between two filters. An identical set of HRC exposures was later obtained in a background field selected to represent the M31 disk+halo contribution to the M32 envelope exposures. The present images

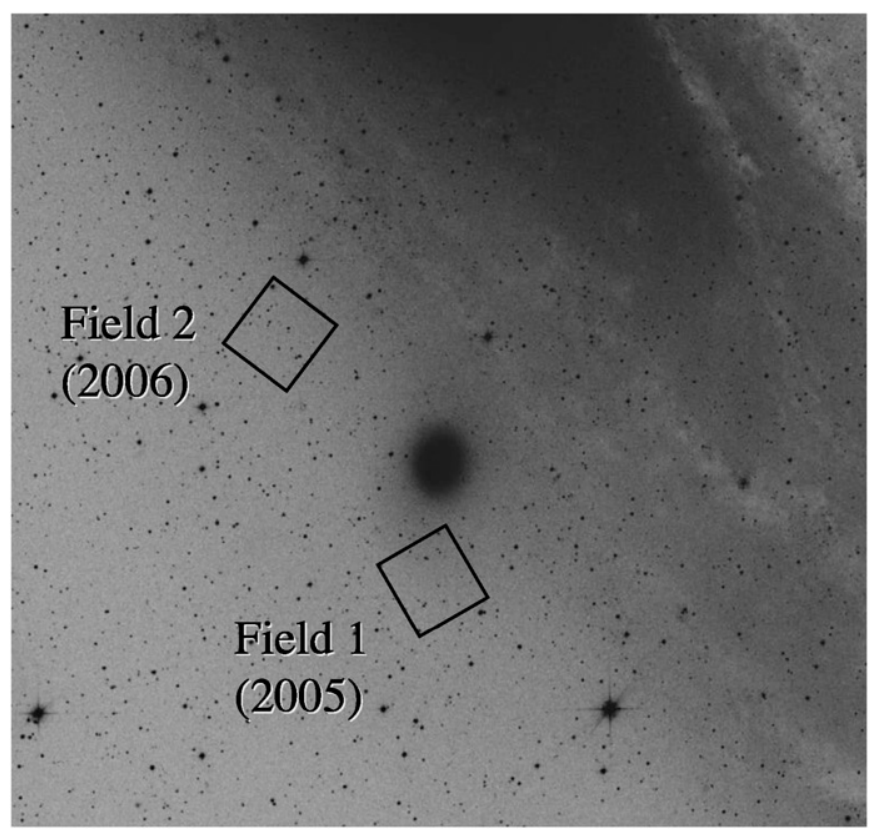

Figure 1. Location of our ACS fields overplotted on a digitized sky survey image in the region of M31. The dwarf elliptical galaxy M32 is near the center of the image. North is up and east is to the left.

are the parallel observations associated with each pointing obtained with the ACS Wide Field Channel (WFC) using the F606W $(\sim V)$ filter. Table 1 provide some details of the observational data. The temporal coverage is 2.2 days for Field 1 and 3.1 days for Field 2.

Figure 1 shows the locations of these fields relative to M31. While the M32-background field was carefully selected to fall along the M31 isophote that ran through the M32 envelope field, the two fields were significantly separated in time, thus different spacecraft rolls between the two epochs caused the parallel WFC aperture to fall randomly about the primary HRC fields. Field 1 thus samples a region that is $4.5 \mathrm{kpc}$ (in a projected distance) from the center of M31, while Field 2 is located $6.6 \mathrm{kpc}$ from the center. Note that throughout this paper, we adopt an M31 distance modulus of $(m-M)_{0}=24.43$ corresponding to a distance of $770 \mathrm{kpc}$ (Freedman \& Madore 1990).

The spacecraft was dithered in a complex pattern to both achieve Nyquist sampling in the HRC and rejection of CCD defects. Each pair of orbits was dithered in a $2 \times 2$ square pattern of 0.5 HRC pixel steps, followed by larger steps to trace a skewed-square spiral of $\sim 0.2$ arcsec total amplitude over the 16 total orbits devoted to each filter/field combination. The subpixel dithering to achieve Nyquist sampling in the HRC is not optimal for the 2 times larger pixels of the WFC, but the larger scale dither pattern fortunately served to offer diverse sampling information for the parallel imagery. 


\section{REDUCTIONS}

\subsection{Photometry of Program Frames}

We chose to work on the FLT images as retrieved from the HST archive. These frames have been bias subtracted and flat fielded, but, unlike the drizzled (DRZ) images, they retain the geometric distortions of ACS (Mahmud \& Anderson 2008). Photometry was performed using the same procedure as Sarajedini et al. (2006). The first step is the application of geometric correction images to the Wide Field Channel 1 and 2 (WFC1 and WFC2, respectively) portions of the FLT images. After this step, the data quality maps are applied where the values of the bad pixels in the science images are set to a number well below the sky background to be sure the photometry software ignores those pixels. At this point, the resultant images are ready to be photometered.

The detection of the stellar profiles and the measurement of magnitudes were done with the DAOPHOT/ALLSTAR/ ALLFRAME crowded-field photometry software (Stetson 1987, 1994). After the application of the standard FIND and PHOT routines to detect stars and perform aperture photometry on them, ALLSTAR was applied to each of the 32 images in order to derive well-determined positions for all of the stars. In this step and in subsequent ones involving the application of a point-spread function (PSF) in order to determine positions and magnitudes, we made use of the high signal-to-noise PSFs constructed by Sarajedini et al. (2006). The reader is referred to that paper for the details of the PSF construction process.

The stellar positions from the ALLSTAR runs were used to construct a coordinate transformation between each of the 32 images and these were used to combine all of the images into one master frame per field. This combined frame was then input into ALLSTAR, from which a master coordinate list of stellar profiles was constructed. The resultant coordinate list along with the spatial transformation between the images and the PSFs were used in ALLFRAME to derive magnitudes for all detected profiles on each image. At this point, the measurements on each of the individual frames were matched and only stars appearing on all 32 images were kept.

The standardization of the individual magnitudes proceeded in the following manner. First, the correction for the charge transfer efficiency problem was applied using the prescription of Reiss \& Mack (2004). The magnitudes were then adjusted to a radius of 0.5 arcsec and corrected for exposure time. Offsets to an infinite radius aperture published by Sirianni et al. (2005) were then applied. Finally, the resultant values were calibrated to the VegaMAG system using the zero point for the F606W filter from Sirianni et al. (2005). A correction to this zero point amounting to 0.022 mag was applied as a result of a revised calibration of the ACS/WFC photometric performance by Mack et al. (2007). Each of our magnitude measurements is affected by three sources of systematic error: the uncertainty in the aperture corrections $( \pm 0.02 \mathrm{mag})$, the error in the correction to infinite aperture $( \pm 0.00 \mathrm{mag})$ for the F606W filter, and the error in the VegaMAG zero point $( \pm 0.02 \mathrm{mag})$.

\subsection{Characterization of the Variable Stars}

For a given star with 32 magnitude measurements, we calculated the mean photometric error as returned by ALLFRAME $\left(\left\langle\sigma_{\text {err }}\right\rangle\right)$ and the standard deviation of the measurements $\left(\sigma_{\mathrm{sd}}\right)$. For the first round of variable searching, we considered any star a candidate if $\sigma_{\mathrm{sd}} /\left\langle\sigma_{\mathrm{err}}\right\rangle \geqslant 3.0$. Approximately 3000 stars fit this criterion in each of our two fields.
These stars were then input into our template-fitting periodfinding algorithm, which is based on the method used by Sarajedini et al. (2006) as originally formulated by Layden \& Sarajedini (2000). We have taken the FORTRAN code written for the Layden \& Sarajedini (2000) study and rewritten it using the Interactive Data Language (IDL) incorporating a graphical user interface (GUI). The original FORTRAN code used the "amoeba" minimum-finding algorithm exclusively, but our code, dubbed FITLC, ${ }^{6}$ has the option to use a more robust algorithm known as "pikaia" which has its roots in the study of genetics. The software uses 10 template light curves-six ab-type RR Lyraes, two c-type RR Lyraes, one eclipsing binary, and one contact binary. It searches over a period range from 0.2 day to a specified maximum (2.2 days for our Field 1 and 3.1 days for Field 2) looking for the period that minimizes the value of $\chi^{2}$. This is accomplished with a two-step process. First pikaia is used to find the combination of epoch, amplitude, and mean magnitude that minimize $\chi^{2}$ at evenly spaced period increments of 0.01 day. Then pikaia is applied again to find the combination of epoch, amplitude, mean magnitude, and period that minimize $\chi^{2}$ within \pm 0.01 day of the period with the lowest $\chi^{2}$. The best fitting period from this final step is taken to be the period of the variable. The resultant phased light curves for each star were visually examined, and the stars that presented a compelling case for variability were retained in our final database. Of the $\sim 6000$ total stars originally fit, 752 exhibit genuine variability as shown by our data.

As a test of our template-fitting method, we have also applied the Lomb-Scargle period-finding algorithm (Scargle 1982; Horne \& Baliunas 1986) to the time series photometry of the RR Lyraes in our sample. We find a mean difference of $0.0007 \mathrm{~d}$ in the periods determined by the two methods throughout the period range of RR Lyraes. In the minority of cases where template-fitting and Lomb-Scargle yield significantly different results, the resultant phased light curves are of significantly higher quality for the former method as compared with the latter. Furthermore, to test for the presence of aliasing effects in our derived periods, we have also examined fitted light curves using periods that correspond to $\chi^{2}$ minima near half of the optimum period. In all cases, these fits are clearly inferior to the ones yielded by the optimum period from FITLC.

Tables 2 and 3 list the individual F606W magnitudes of each variable at each epoch wherein $2,450,000$ has been subtracted from the epoch value, while Tables 4 and 5 list the candidate variables in our two fields along with their properties such as period, amplitude, and mean intensity-weighted magnitude. These stars fall into two broad categories. First, there are those that clearly show variability, but their periods are comparable to or longer than our observing window. These are referred to as "long period" in Tables 4 and 5. There is also one candidate anomalous Cepheid in our data set, which is so indicated in Table 5. The second category includes stars that exhibit clear periodic variability with a period that is significantly shorter than our observing window but longer than $0.2 \mathrm{~d}$. These are the stars for which we can be confident of our periods. Some examples of stars with periods longer than our observing window are shown in Figure 2, while Figure 3 shows phased light curves of a number of contact and eclipsing binaries in our data set. Figure 4 displays the phased light curves of all of the RR Lyrae variables for which we have derived periods.

\footnotetext{
6 http://www.astro.ufl.edu/ cmancone/fitlc.html
} 
Table 2

Raw Light Curves Field 1

\begin{tabular}{|c|c|c|c|c|c|c|c|c|c|c|}
\hline Epoch & $001 \mathrm{Mag}$ & 001 Err & $002 \mathrm{Mag}$ & 002 Err & 003 Mag & 003 Err & 004 Mag & 004 Err & $005 \mathrm{Mag}$ & $005 \mathrm{Err}$ \\
\hline 3635.97534180 & 21.414 & 0.022 & 21.960 & 0.021 & 24.033 & 0.033 & 24.782 & 0.059 & 24.639 & 0.049 \\
\hline 3635.99096680 & 21.417 & 0.024 & 21.984 & 0.017 & 24.023 & 0.033 & 24.826 & 0.052 & 24.664 & 0.049 \\
\hline 3636.05566406 & 21.400 & 0.028 & 22.032 & 0.025 & 24.011 & 0.021 & $\ldots$ & $\cdots$ & 24.650 & 0.052 \\
\hline 3636.10693359 & 21.405 & 0.018 & 22.051 & 0.030 & 24.016 & 0.051 & 24.750 & 0.034 & 24.604 & 0.045 \\
\hline 3636.12304688 & 21.384 & 0.030 & 22.083 & 0.027 & 24.013 & 0.042 & 24.582 & 0.038 & 24.585 & 0.046 \\
\hline 3636.18969727 & 21.384 & 0.025 & 22.118 & 0.028 & $\cdots$ & $\cdots$ & $\cdots$ & $\cdots$ & 24.607 & 0.056 \\
\hline 3636.70800781 & 21.329 & 0.011 & 22.305 & 0.027 & 24.050 & 0.059 & 24.706 & 0.039 & 24.890 & 0.037 \\
\hline 3636.72363281 & 21.321 & 0.016 & 22.332 & 0.036 & 24.036 & 0.036 & 24.755 & 0.031 & 24.799 & 0.042 \\
\hline 3636.77294922 & 21.323 & 0.010 & 22.382 & 0.019 & $\cdots$ & $\cdots$ & 24.853 & 0.044 & 24.617 & 0.022 \\
\hline 3636.78833008 & 21.315 & 0.015 & 22.339 & 0.029 & 24.037 & 0.035 & 24.846 & 0.048 & 24.552 & 0.049 \\
\hline 3637.77368164 & 21.222 & 0.014 & 21.832 & 0.022 & 23.992 & 0.040 & 24.629 & 0.037 & 24.619 & 0.025 \\
\hline 3637.78930664 & 21.198 & 0.016 & 21.805 & 0.021 & 24.012 & 0.022 & 24.553 & 0.036 & 24.640 & 0.026 \\
\hline 3637.83862305 & 21.197 & 0.018 & 21.538 & 0.033 & 23.949 & 0.030 & 24.672 & 0.039 & 24.643 & 0.040 \\
\hline 3637.85424805 & 21.209 & 0.011 & 21.542 & 0.023 & 23.994 & 0.037 & 24.704 & 0.048 & 24.649 & 0.045 \\
\hline 3637.90551758 & 21.225 & 0.020 & 21.477 & 0.027 & 23.980 & 0.034 & 24.932 & 0.071 & 24.646 & 0.098 \\
\hline 3637.92163086 & 21.192 & 0.021 & 21.498 & 0.028 & 23.977 & 0.030 & 24.875 & 0.050 & 24.617 & 0.062 \\
\hline 3637.97216797 & 21.208 & 0.022 & 21.591 & 0.026 & $\cdots$ & $\cdots$ & 24.882 & 0.026 & 24.606 & 0.087 \\
\hline 3637.98803711 & 21.208 & 0.014 & 21.541 & 0.023 & 23.987 & 0.031 & 24.841 & 0.044 & $\cdots$ & $\cdots$ \\
\hline 3638.04028320 & 21.183 & 0.017 & 21.634 & 0.018 & 24.000 & 0.025 & 24.764 & 0.032 & 24.669 & 0.042 \\
\hline 3638.05566406 & 21.180 & 0.020 & 21.655 & 0.025 & 24.007 & 0.025 & 24.673 & 0.050 & 24.594 & 0.071 \\
\hline
\end{tabular}

All of the stars that exhibit RR Lyrae light curves also have apparent magnitudes in the range one would expect if they are at the distance of M31. Therefore, it is reasonable to assume that most if not all of these objects are RR Lyrae stars belonging to M31 and/or its environs. This assertion will become clearer when we compare the luminosity function (LF) of the nonvariable stars with that of the RR Lyraes.

It should be noted that we are much less confident about the properties of the eclipsing and contact binaries that we have identified as compared with the RR Lyraes. This is because we know what period range to expect for the RR Lyraes $(\sim 0.25 \mathrm{~d}$ to $\sim 0.90 \mathrm{~d}$ ), so that our observing window provides coverage of multiple cycles of variation for a given RR Lyrae star. In contrast, the periods of the eclipsing and contact binaries cannot be similarly constrained, so it is difficult for us to ensure that our observing window is sufficient to derive the periods of these variables. As such, we have provided information for these stars (positions, periods, amplitudes, and magnitudes) so that future observers can confirm the nature of their variability, but we will not consider them further here. Instead, for the remainder of this paper, we will limit our discussion to the 681 RR Lyrae variables in our sample and what they reveal about the properties of the M31 system.

\subsection{Light Curve Simulations}

In order to characterize the possible biases in the derived periods of our variable star sample, we have performed simulations of our light-curve-fitting technique in the following manner. For the ab-type RR Lyraes, we selected one of the light curve templates (the results are insensitive to the actual ab-type template used) and produced artificial variables with a period range of $0.45-0.80$ days and amplitudes between 0.3 and $1.3 \mathrm{mag}$. For the c-type RR Lyraes, a period range of $0.25-0.40$ days and an amplitude range of $0.2-0.5 \mathrm{mag}$ were used. One thousand variables were generated in each case, and the mean photometric error at the level of the RR Lyraes was used to populate the light curves using the same observing window as the actual data. These simulated light curves were input into our template lightcurve-fitting software. We are interested in comparing the input periods with the output periods in order to gauge any possible biases present in our analysis method.

Figures 5 (RRab) and $6(\mathrm{RRc})$ show the result of these fits for Field 1 wherein the upper panel shows the mean difference between the input and output periods, while the lower panel illustrates the differences in the period distributions. The results of the simulations for RR Lyraes in Field 2 are indistinguishable from those in Field 1. Of the 1000 ab-type RR Lyraes generated, none were mistaken for any other type of variable among the 10 light curve templates used in the fitting. For the c-type light curves, two of the 1000 generated variables were fit with a contact binary template. We find no significant biases in our determination of the periods for both types of RR Lyraes. As such, we will not apply any sort of correction to our derived periods. As for the errors in the period and amplitude determinations, these simulations suggest that an individual abtype RR Lyrae has an error of \pm 0.005 day and \pm 0.044 mag, 
Table 3

Raw Light Curves Field 2

\begin{tabular}{|c|c|c|c|c|c|c|c|c|c|c|}
\hline Epoch & $366 \mathrm{Mag}$ & 366 Err & $367 \mathrm{Mag}$ & 367 Err & $368 \mathrm{Mag}$ & 368 Err & $369 \mathrm{Mag}$ & 369 Err & $370 \mathrm{Mag}$ & $370 \mathrm{Err}$ \\
\hline 3775.87866211 & 21.896 & 0.034 & 22.464 & 0.029 & 22.360 & 0.038 & & 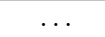 & 23.676 & 0.023 \\
\hline 3775.89428711 & 21.955 & 0.031 & 22.409 & 0.050 & 22.387 & 0.038 & 23.583 & 0.025 & 23.667 & 0.036 \\
\hline 3775.95971680 & 21.885 & 0.035 & 22.443 & 0.029 & 22.342 & 0.038 & 23.626 & 0.026 & 23.664 & 0.030 \\
\hline 3776.01098633 & 21.930 & 0.028 & 22.417 & 0.043 & 22.347 & 0.029 & 23.686 & 0.027 & 23.607 & 0.020 \\
\hline 3776.02685547 & 21.881 & 0.034 & 22.400 & 0.032 & 22.387 & 0.032 & 23.689 & 0.036 & 23.579 & 0.036 \\
\hline 3776.09350586 & 21.914 & 0.019 & 22.323 & 0.063 & 22.332 & 0.028 & 23.688 & 0.037 & 23.592 & 0.022 \\
\hline 3777.54418945 & 21.748 & 0.044 & 22.178 & 0.041 & 22.234 & 0.034 & 23.806 & 0.070 & 23.671 & 0.035 \\
\hline 3777.55957031 & 21.693 & 0.035 & 22.174 & 0.054 & 22.178 & 0.030 & 23.807 & 0.063 & 23.741 & 0.034 \\
\hline 3777.60913086 & 21.706 & 0.034 & 22.179 & 0.049 & 22.159 & 0.029 & 23.836 & 0.053 & 23.740 & 0.041 \\
\hline 3777.62475586 & 21.722 & 0.043 & 22.174 & 0.040 & 22.053 & 0.060 & 23.872 & 0.066 & 23.707 & 0.034 \\
\hline 3778.41113281 & 21.662 & 0.028 & 22.085 & 0.020 & 22.113 & 0.024 & 23.397 & 0.020 & 23.635 & 0.055 \\
\hline 3778.47485352 & 21.656 & 0.032 & 22.074 & 0.020 & 22.128 & 0.033 & 23.454 & 0.029 & 23.702 & 0.061 \\
\hline 3778.54150391 & 21.652 & 0.033 & 22.067 & 0.026 & 22.113 & 0.035 & 23.553 & 0.036 & 23.899 & 0.056 \\
\hline 3778.55712891 & 21.636 & 0.032 & 22.065 & 0.024 & 22.097 & 0.023 & 23.515 & 0.026 & 23.905 & 0.059 \\
\hline 3778.60839844 & 21.589 & 0.043 & 22.055 & 0.034 & 22.081 & 0.035 & 23.567 & 0.030 & 23.981 & 0.032 \\
\hline 3778.62451172 & 21.614 & 0.025 & 22.052 & 0.039 & 22.085 & 0.033 & 23.594 & 0.029 & 23.941 & 0.027 \\
\hline 3778.67504883 & 21.618 & 0.032 & 22.055 & 0.035 & 22.087 & 0.030 & 23.584 & 0.023 & 23.783 & 0.038 \\
\hline 3778.69116211 & 21.599 & 0.044 & 22.045 & 0.035 & 22.059 & 0.042 & 23.632 & 0.034 & 23.834 & 0.034 \\
\hline 3778.74340820 & 21.592 & 0.028 & 22.056 & 0.038 & 22.077 & 0.036 & 23.671 & 0.023 & 23.735 & 0.026 \\
\hline 3778.75903320 & 21.609 & 0.034 & 22.041 & 0.031 & 22.054 & 0.059 & 23.721 & 0.025 & 23.815 & 0.040 \\
\hline
\end{tabular}

Table 4

Field 1 Variable Stars

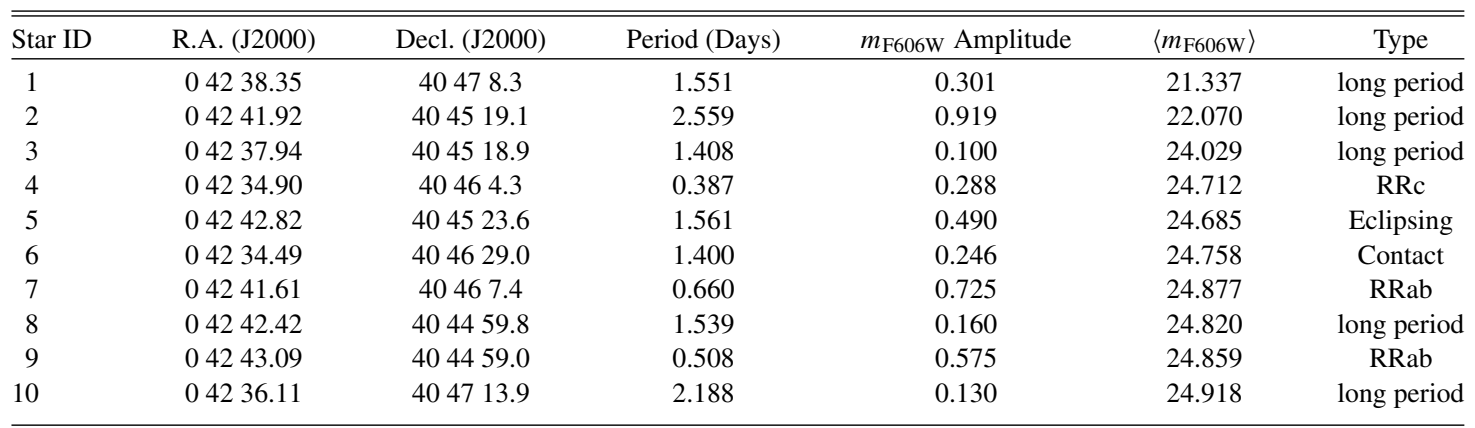

(This table is available in its entirety in machine-readable and Virtual Observatory (VO) forms in the online journal. A portion is shown here for guidance regarding its form and content.)

respectively. For a c-type RR Lyrae star, these error values are \pm 0.001 day and $\pm 0.022 \mathrm{mag}$.

\section{RESULTS}

\subsection{Luminosity Functions}

Figure 7 displays a comparison of the LFs of the nonvariable stars in the two fields. Both distributions feature a quick rise from brighter magnitudes to fainter ones with a pronounced peak at $m_{\mathrm{F} 606 \mathrm{~W}} \sim 25.3$ representing the core-helium burning horizontal branch (HB) stars. A sudden drop in both LFs at $m_{\mathrm{F} 606 \mathrm{~W}} \sim 27.7$ suggests the onset of significant incompleteness as the limit of the photometry is approached. The fact that the HB stars are more than 2 magnitudes brighter than this completeness threshold indicates that our sample of RR Lyrae variable candidates should not be adversely biased by photometric incompleteness.

The two panels of Figure 8 compare the LF of the nonvariable stars with those of the RR Lyraes in the two observed fields. We have used the intensity-weighted magnitudes listed in Tables 4 and 5 to construct these distributions. Gaussian fits to the regions around the RR Lyrae LF peaks yield $\left\langle m_{\mathrm{F} 606 \mathrm{~W}}\right\rangle=25.20 \pm 0.04$ for Field 1 and $\left\langle m_{\mathrm{F} 606 \mathrm{~W}}\right\rangle=25.22 \pm 0.04$ for Field 2, where the errors represent the standard error of the mean combined with the uncertainty in the photometric zero point added in quadrature. For the nonvariable stars, these peaks correspond 
Table 5

Field 2 Variable Stars

\begin{tabular}{|c|c|c|c|c|c|c|}
\hline$\underline{\text { Star ID }}$ & R.A. (J2000) & Decl. (J2000) & Period (Days) & $m_{\mathrm{F} 606 \mathrm{~W}}$ Amplitude & $\left\langle m_{\mathrm{F} 606 \mathrm{~W}}\right\rangle$ & Type \\
\hline 366 & 04317.55 & 405842.3 & 1.886 & 0.314 & 21.732 & long period \\
\hline 367 & 04320.31 & 405812.1 & 2.234 & 0.387 & 22.141 & long period \\
\hline 369 & 04324.57 & 405651.9 & 1.366 & 0.501 & 23.625 & long period \\
\hline 370 & 04316.06 & 405829.2 & 1.978 & 0.291 & 23.675 & Eclipsing \\
\hline 371 & 04323.53 & 40573.9 & 0.557 & 0.558 & 23.794 & $\mathrm{RRab}$ \\
\hline 373 & 04317.54 & 405848.8 & 1.142 & 0.839 & 24.299 & Eclipsing \\
\hline 374 & 04320.14 & 405730.7 & 2.570 & 0.368 & 24.386 & Contact \\
\hline 375 & 04320.79 & 405727.2 & 0.600 & 0.563 & 24.454 & $\mathrm{RRab}$ \\
\hline
\end{tabular}

(This table is available in its entirety in machine-readable and Virtual Observatory (VO) forms in the online journal. A portion is shown here for guidance regarding its form and content.)
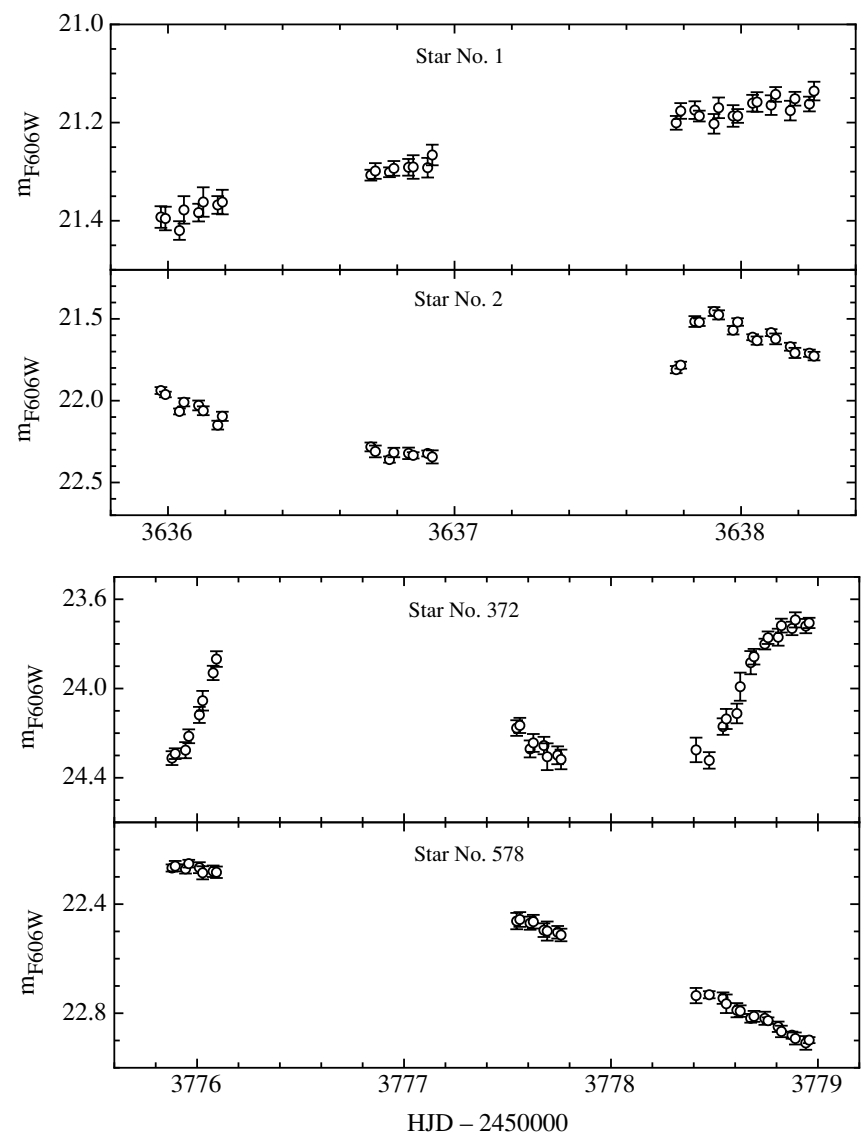

Figure 2. Unphased light curves for a sample of our variable stars with periods that are comparable to or longer than our observing window. The numbers refer to the stars in Tables 4 and 5.

to $\left\langle m_{\mathrm{F} 606 \mathrm{~W}}\right\rangle=25.25 \pm 0.04$ for Field 1 and $\left\langle m_{\mathrm{F} 606 \mathrm{~W}}\right\rangle=$ $25.23 \pm 0.04$ for Field 2 . The 1- $\sigma$ width of these distributions is $\sim 0.11 \mathrm{mag}$. These numbers suggest no significant difference in the mean magnitudes of the RR Lyraes and the nonvariable stars. This serves to confirm our assertion that most if not all of the variable stars in this magnitude range are RR Lyrae variables. In addition, when we combine the RR Lyrae variables from both fields, we find a mean magnitude of $m_{\mathrm{F} 606 \mathrm{~W}}=25.21 \pm 0.01$ on the VEGAmag system. The quoted uncertainty represents the standard error of the mean. When converted to the $V$ band using the mean offset for RR Lyraes in the middle of the instability strip from B2004 of $V-m_{\mathrm{F} 606 \mathrm{~W}}=0.08 \pm 0.04$, we derive

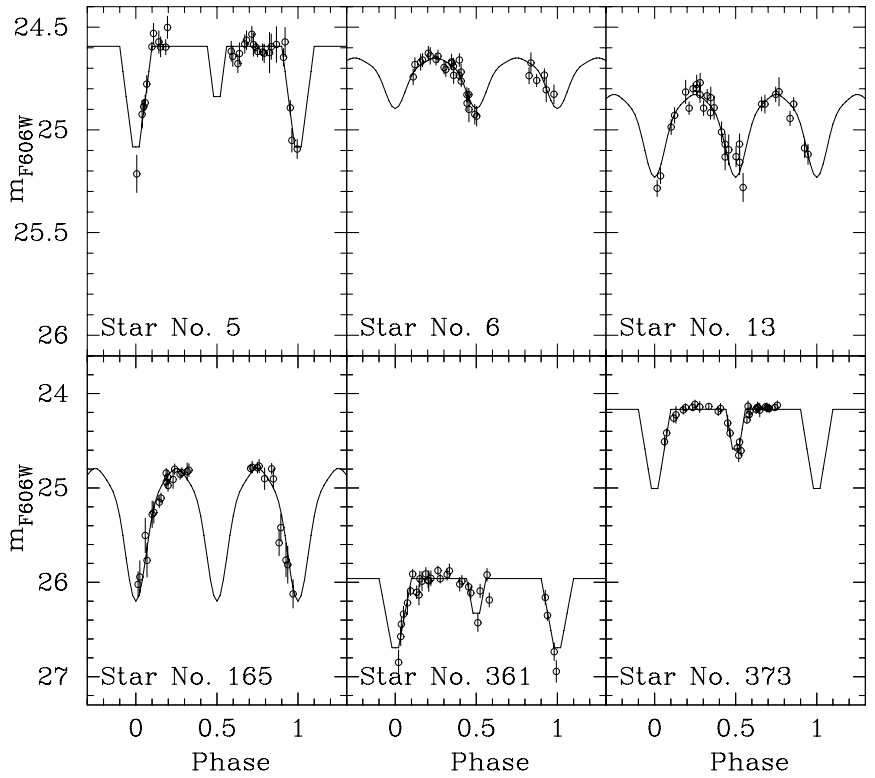

Figure 3. Phased light curves for some of the contact and eclipsing binaries identified in this study.

$\langle V(R R)\rangle=25.29 \pm 0.01$ (random) \pm 0.05 (systematic). This compares favorably with the value of $\langle V(R R)\rangle=25.30 \pm 0.01$ based on the average for the ab-type and c-type RR Lyraes from the B2004 study (see Figure 8). We note in passing that a Gaussian fit to the LF of the B2004 RR Lyraes yields a $1 \sigma$ width of $0.12 \mathrm{mag}$, which is comparable to the value for the RR Lyraes in the present study.

\subsection{Number Ratios}

Of the 681 total RR Lyrae stars in our sample, 555 (267 in Field 1 and 288 in Field 2) are of the ab-type with the remainder being c-type (57 in Field 1 and 69 in Field 2). This leads to a ratio of $N_{\mathrm{c}} / N_{\mathrm{abc}}=0.19 \pm 0.02$, which is quite different than the value of $N_{\mathrm{c}} / N_{\mathrm{abc}}=0.46 \pm 0.11$ inferred from the B2004 data. Therefore, our samples of ab-type and c-type RR Lyraes are a factor of $\sim 2$ greater and a factor of $\sim 2$ less, respectively, than what we would expect based on the B2004 field. Taken at face value, this suggests that the old population in the environs of M31 exhibits different pulsation properties at 4-6 kpc as compared with $11 \mathrm{kpc}$.

To evaluate the validity of this assertion, we need to address the question of incompleteness in our sample of RR 


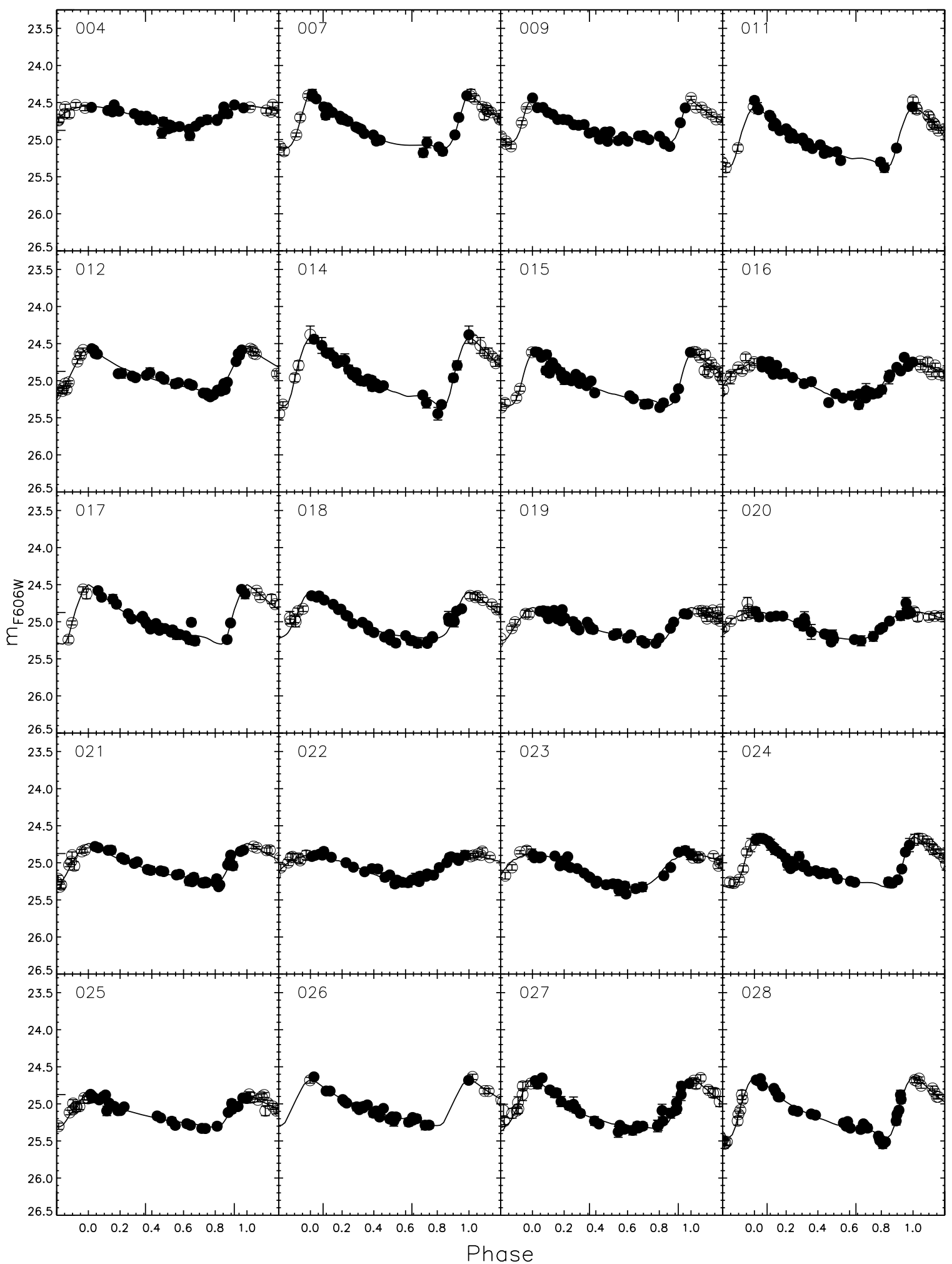

Figure 4. Phased light curves for the 681 RR Lyrae variables identified in this study. The open circles are repeated to complete the light curve for phase less than zero and greater than 1 .

(The complete figure set (35 images) is available in the online journal.)

Lyraes. Are there significant numbers of RR Lyraes in our fields that we have failed to identify? We begin by noting that B2004 claim that their samples of c-type and ab-type RR Lyraes are complete and not significantly contaminated by dwarf Cepheids. Therefore, we can gain some insight by comparing the amplitude distributions of the B2004 RR Lyrae variables with our sample as shown in Figure 9. This comparison suggests that the amplitude distribution of the B2004 RR Lyraes is consistent with those of our sample. Application of the Kolmogorov-Smirnov test to these distributions confirms this 


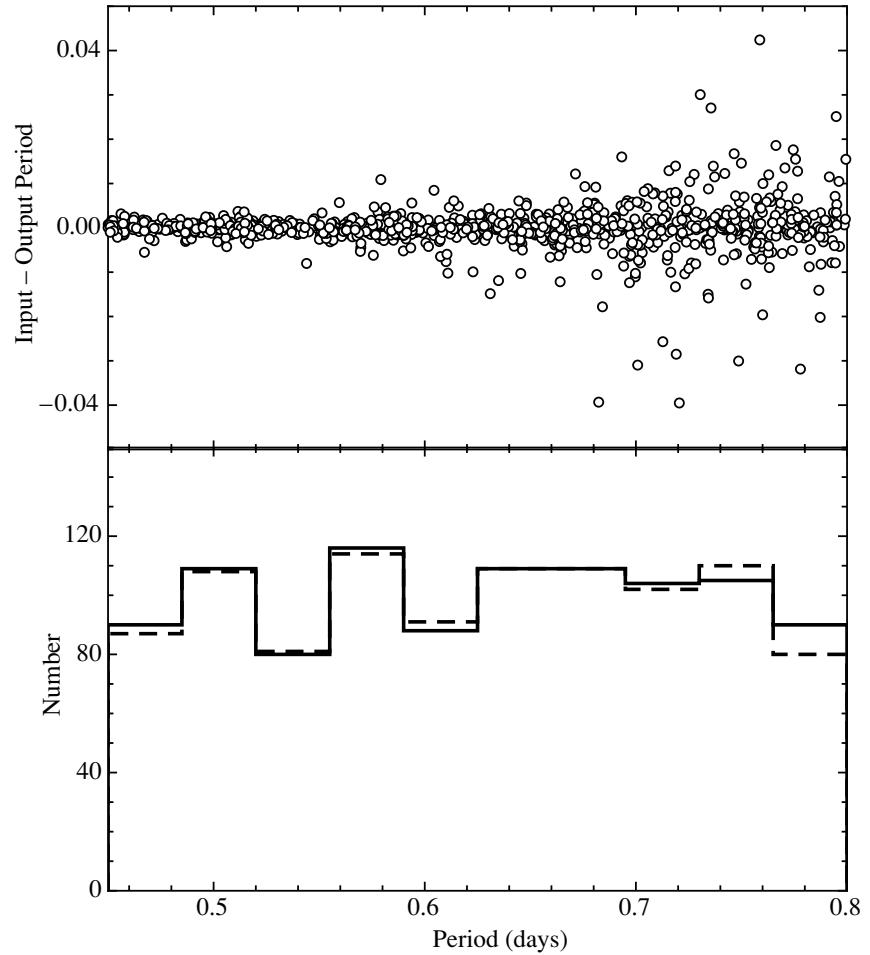

Figure 5. Results of the light curve simulations performed in order to characterize any biases in our period-finding algorithm in the case of the RRab variables. The upper panel plots the variation of input minus recovered period, while the lower panel plots the period distribution both as a function of period in days. The solid line is the input distribution, while the dashed line represents the recovered one. These simulations suggest that the combination of the input data and the period-finding algorithm does not introduce significant biases in our derived periods.

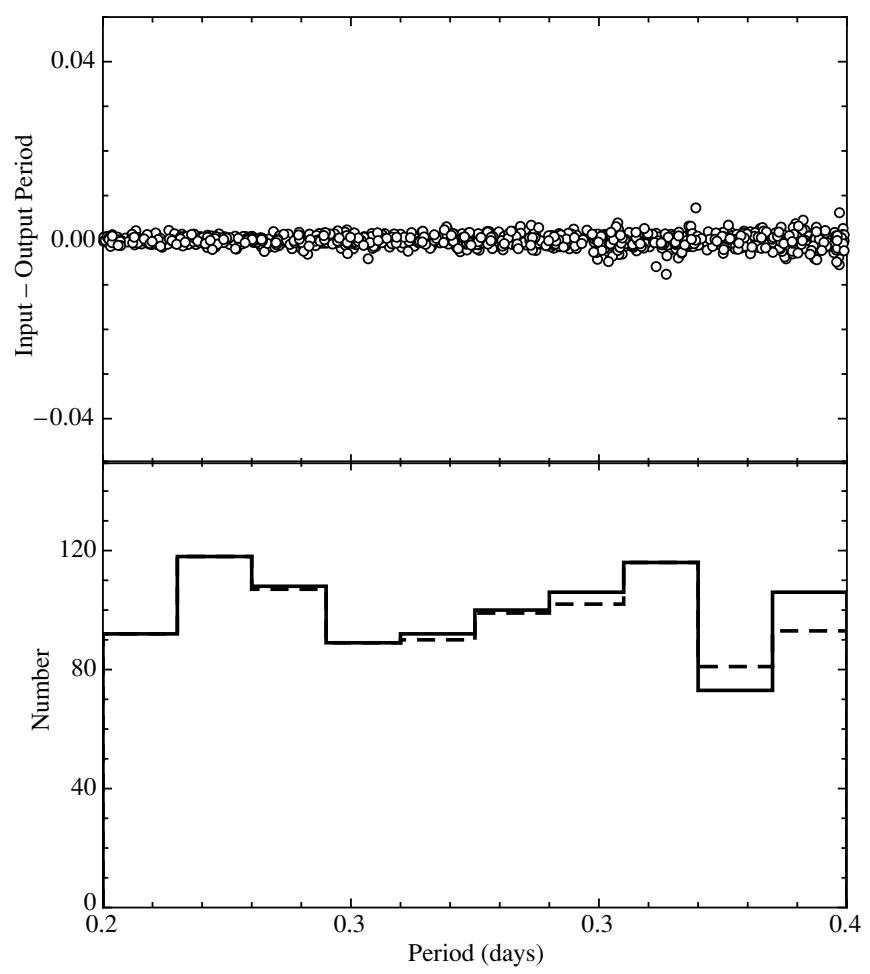

Figure 6. Same as Figure 6 except that the simulations have been performed using an c-type RR Lyrae variable light curve.

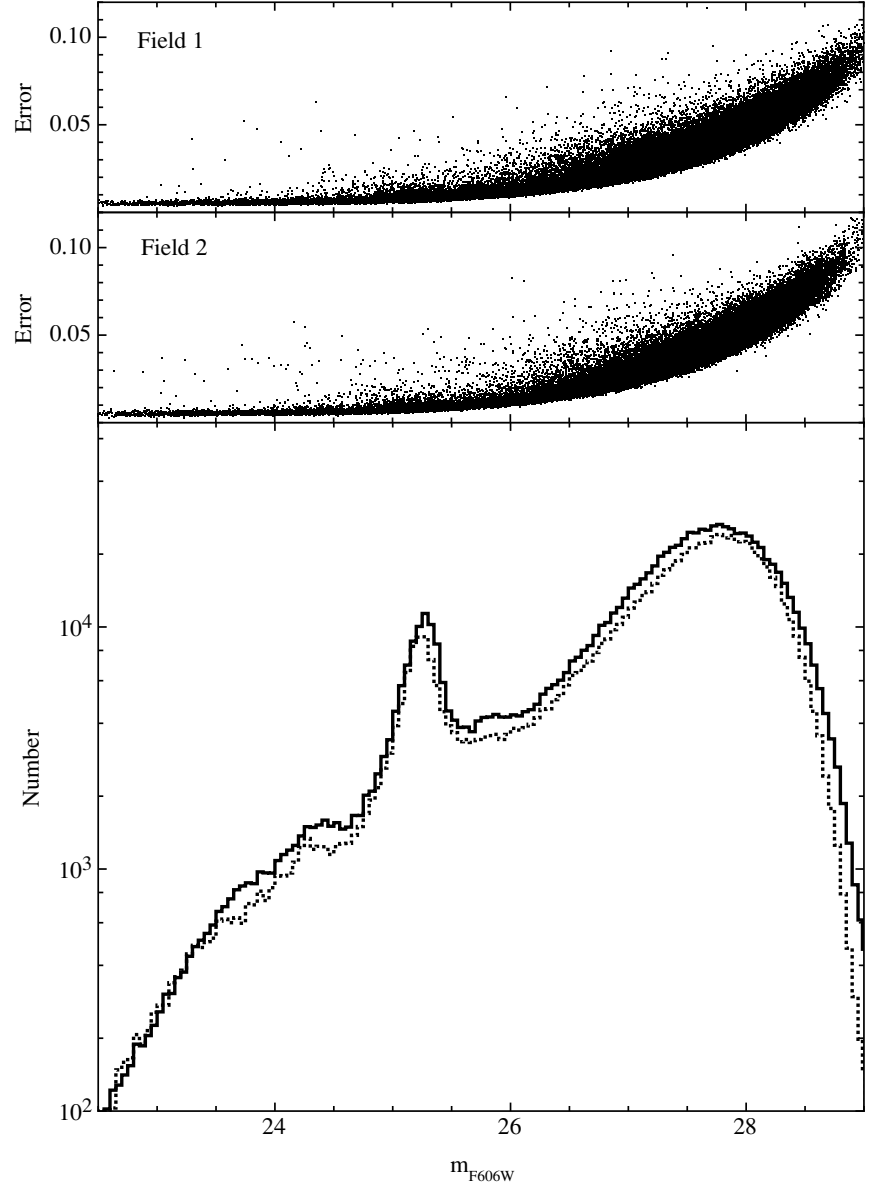

Figure 7. Luminosity functions for the nonvariable stars in the two fields studied herein. The solid line in the lower panel is the F606W band LF for the field observed in 2005 (field 1 in Table 1), while the dashed line is the LF for the region observed in 2006 (field 2 in Table 1). The two upper panels show the variation of the photometric error as output by ALLFRAME with magnitude. We have plotted every 10th point to make the appearance of these plots manageable.

suggestion. The fact that, at the low-amplitude end, the two distributions are not substantially different argues that significant numbers of low-amplitude RR Lyraes are not missing from our sample.

Another possibility of explaining the differences in the $N_{\mathrm{c}} / N_{\mathrm{abc}}$ ratio between our fields and the one at $11 \mathrm{kpc}$ is that our Field 1 data are significantly influenced by the stellar populations of M32 and not M31. In fact, we find $N_{\mathrm{c}} / N_{\mathrm{abc}}=$ $0.18 \pm 0.0 .025$ in Field 1 and $N_{\mathrm{c}} / N_{\mathrm{abc}}=0.19 \pm 0.025$ in Field 2, which are statistically indistinguishable from each other. In addition, the density of RR Lyrae variables and their period distributions are indistinguishable between Fields 1 and 2. For example, for the ab-type variables, Field 1 exhibits a mean period of $\left\langle P_{\mathrm{ab}}\right\rangle=0.553 \pm 0.004$, while Field 2 shows $\left\langle P_{\mathrm{ab}}\right\rangle=0.561 \pm 0.005$. In the case of the c-type RR Lyraes, the analogous values are $\left\langle P_{\mathrm{c}}\right\rangle=0.326 \pm 0.005$ and $\left\langle P_{\mathrm{c}}\right\rangle=0.327 \pm 0.004$, respectively. These values suggest that both of our fields sample the regions around M31 and are minimally contaminated by M32 RR Lyraes.

If this difference in the $N_{\mathrm{c}} / N_{\mathrm{abc}}$ ratio between a radial distance of $11 \mathrm{kpc}$ and $4-6 \mathrm{kpc}$ in M31 is real, then it suggests that the RR Lyraes at $11 \mathrm{kpc}$ are more akin to their brethren in Oosterhoff type II globular clusters, while those at 4-6 kpc are more like RR Lyraes in Oosterhoff type I clusters. This is based on the fundings of Castellani et al. (2003) who examined the 


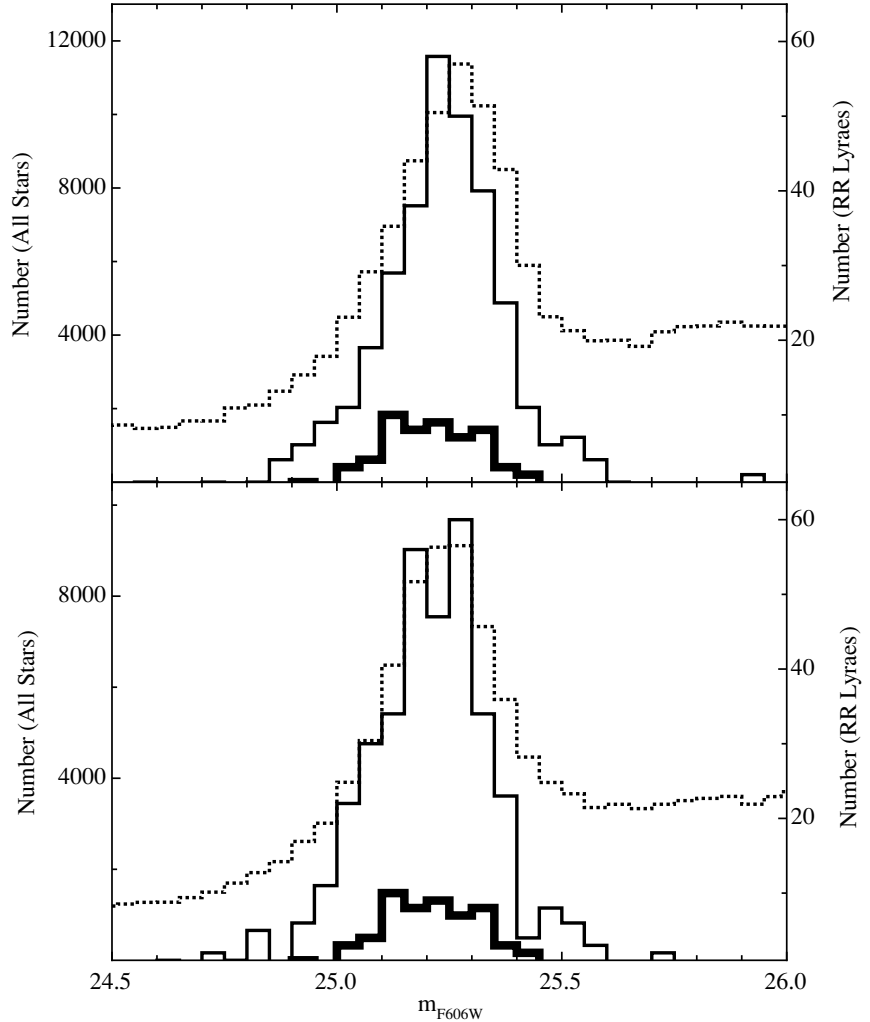

Figure 8. Luminosity functions for the nonvariable stars (dashed lines) compared with those of the RR Lyrae candidates (thin solid lines) in the two fields studied herein (see Table 1). The thicker solid lines represent the RR Lyrae stars from the study of Brown et al. (2004).

$N_{\mathrm{c}} / N_{\mathrm{abc}}$ ratio in Galactic globulars. They found that among the 12 clusters with 40 or more variables, $\left\langle N_{\mathrm{c}} / N_{\mathrm{abc}}\right\rangle=0.37$ for Oosterhoff II clusters and $\left\langle N_{\mathrm{c}} / N_{\mathrm{abc}}\right\rangle=0.17$ for those of Oosterhoff type I. These compare favorably with the values of $\left\langle N_{\mathrm{c}} / N_{\mathrm{abc}}\right\rangle=0.46$ for the $11 \mathrm{kpc}$ field and $\left\langle N_{\mathrm{c}} / N_{\mathrm{abc}}\right\rangle=0.19$ for the 4-6 kpc field.

\subsection{Periods, Amplitudes, and Metallicities}

The Bailey diagram for our sample of RR Lyraes is shown in Figure 10 where we plot the variables in the two fields using different colors. However, it is clear that they occupy the same regions of this diagram. The ab-type RR Lyrae variables are shown with open circles, while the c-type stars are plotted as open triangles. The dashed line in Figure 10 represents the relation exhibited by the RRab stars in the B2004 field. The solid lines are the relations for Oosterhoff I and II globular clusters from Clement (2000). These lines have been adjusted to account for the difference between an amplitude in the $V$ band and one in the F606W band. Interestingly, the B2004 RR Lyrae relation is closer to the OoI line, even though the $N_{\mathrm{c}} / N_{\mathrm{abc}}$ ratio in the B2004 field is closer to that of OoII clusters. It is unclear why this should be the case.

The B2004 line appears to be offset compared with our data suggesting slightly shorter periods for the RRab variables in our fields. This behavior is further exemplified in Figure 11 which shows the period distributions in the two fields compared with the mean periods of the ab- and c-type RR Lyraes from B2004. We find mean periods of $\left\langle P_{\mathrm{ab}}\right\rangle=0.557 \pm 0.003$ and $\left\langle P_{\mathrm{c}}\right\rangle=0.327 \pm 0.003$. For the $\mathrm{B} 2004$ field, these values are $\left\langle P_{\mathrm{ab}}\right\rangle=0.594 \pm 0.015$ and $\left\langle P_{\mathrm{c}}\right\rangle=0.316 \pm 0.007$. The mean periods of the c-type variables are statistically indistinguishable
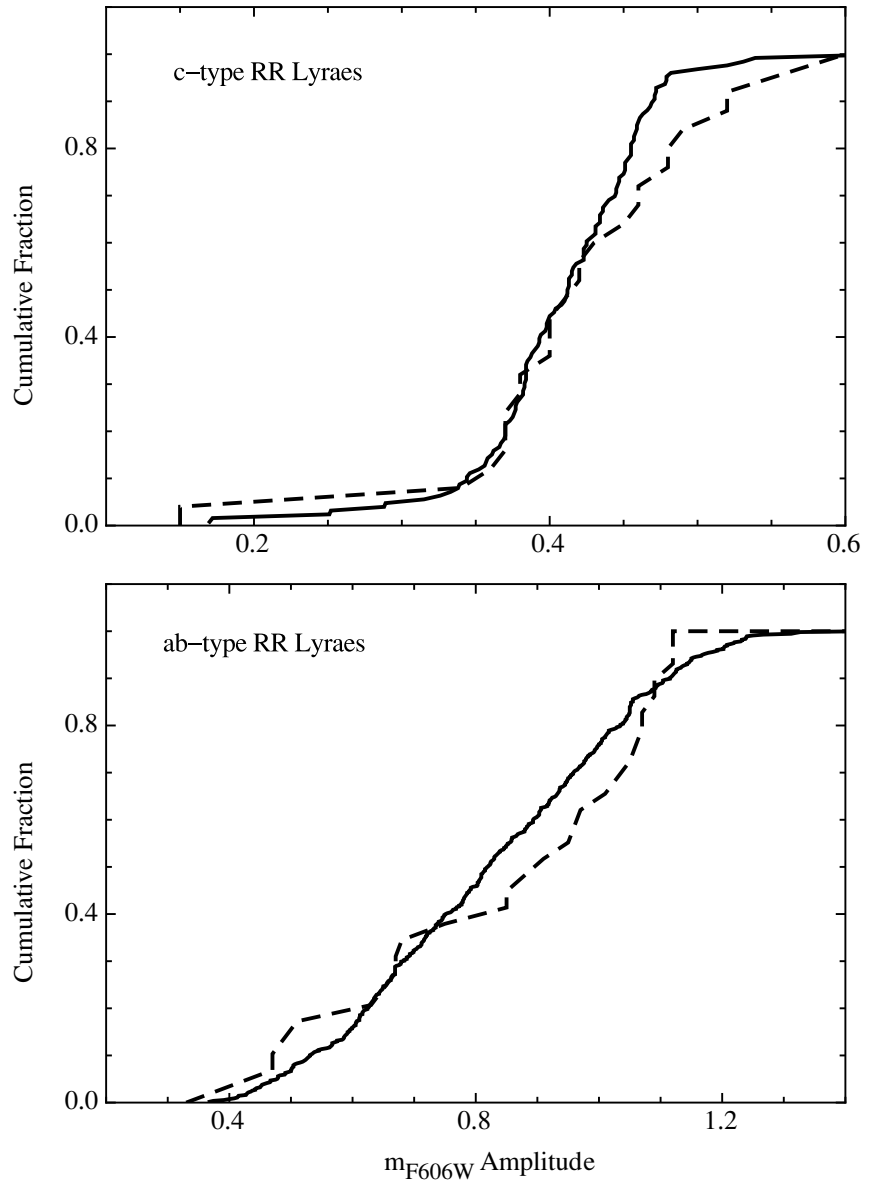

Figure 9. Upper panel shows the amplitude distribution of the c-type RR Lyraes from the present study (solid line) and the work of Brown et al. (2004, dotted line) scaled to the same maximum. The lower panel is the same as the upper one except that the ab-type RR Lyraes are plotted.

from each other, but the ab-types in our fields exhibit a somewhat shorter period as compared with those in the B2004 fields.

It is well known that as the metallicity of ab-type RR Lyraes increases, their periods decrease (e.g., Sandage 1993; Layden 1995; Sarajedini et al. 2006). Thus, the period distribution of these stars (Figure 11) can be converted to a metallicity distribution using equations derived by previous investigators. Using the data of A. C. Layden (2005, private communication) for 132 Galactic RR Lyraes in the solar neighborhood, Sarajedini et al. (2006) established a relation between period and metal abundance of the form

$$
[\mathrm{Fe} / \mathrm{H}]=-3.43-7.82 \log P_{\mathrm{ab}}
$$

This equation does not take into account the amplitudes of the RR Lyraes even though, as Figure 10 shows, there is a relation between amplitude and period for the ab-types. The work of Alcock et al. (2000) yielded a period-amplitude-metallicity relation of the form

$$
[\mathrm{Fe} / \mathrm{H}]=-8.85\left[\log P_{\mathrm{ab}}+0.15 A(V)\right]-2.60,
$$

where $A(V)$ represents the amplitude in the $V$ band. We applied an $8 \%$ increase to the $m_{\mathrm{F} 606 \mathrm{~W}}$ amplitudes to convert them to $V$ band values (B2004). Figure 12 shows the metallicity distribution function (MDF) for the ab-type RR Lyraes. The top panel compares the results obtained using Equations (1) and 


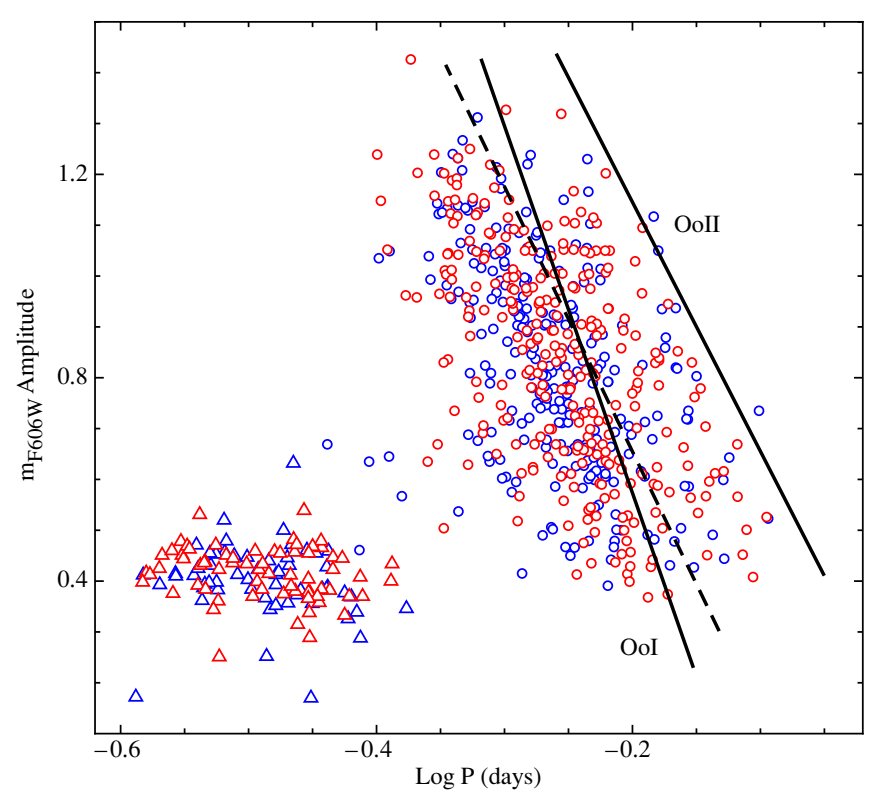

Figure 10. Bailey diagram for the RR Lyrae candidates in our fields. The open circles are the ab-type RR Lyraes, while the open triangles represent the c-type variables. The color coding represents the field number with blue being stars observed in field 1 and red those found in field 2 . This plot shows that while the ab- and c-type RR Lyraes occupy their characteristic locations in this diagram, there is no significant difference between the RR Lyraes in the two observed fields. The dashed line shows the relation exhibited by the RRab stars in the field observed by Brown et al. (2004). The solid lines are the relations for Oosterhoff type I and II globular clusters from Clement (2000). These lines have been adjusted to account for the difference between an amplitude in the $V$ band and one in the F606W band.

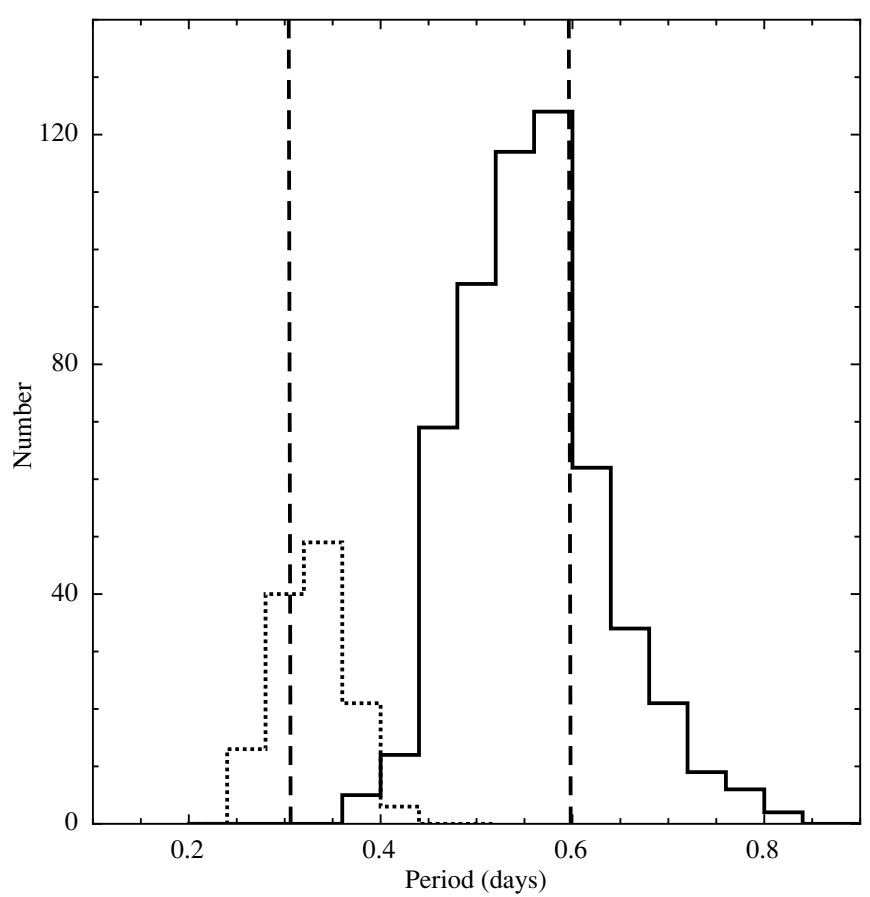

Figure 11. Period distributions of the ab-type (solid) and c-type (dotted) RR Lyrae variables. The dashed vertical lines represent the mean periods of these same stars from Brown et al. (2004). While the mean periods of the c-type variables agree between our study and that of Brown et al. (2004), the mean period of the ab-type RR Lyraes is somewhat shorter in our sample as compared with that of Brown et al.

(2), while the lower panel compares the MDFs for the two fields using Equation (2).

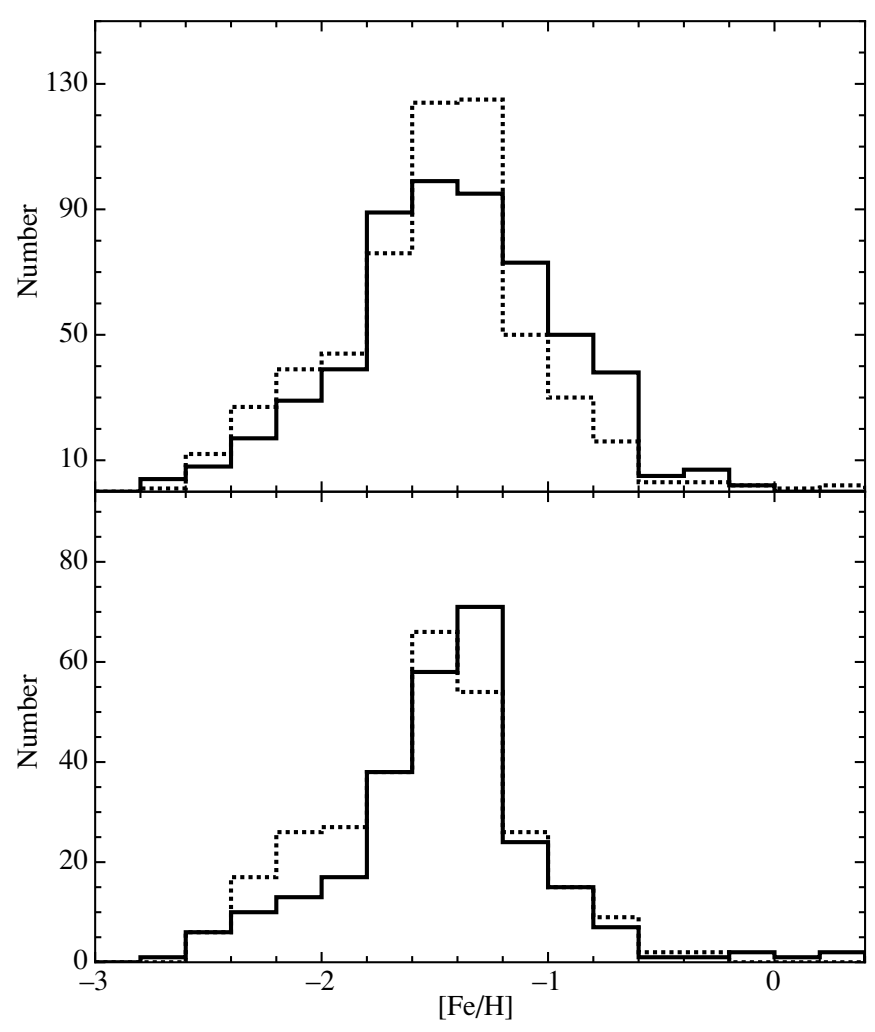

Figure 12. Upper panel shows the metallicity distribution function for our sample of RRab variables using two different formulations for the conversion between period and metal abundance (solid line-Equation (1), dashed lineEquation (2)). The lower panel shows the MDFs derived using Equation (2) for the two observed fields (dashed line-Field 2, solid line-Field 1).

We see in the upper panel of Figure 12 that, while the two MDFs exhibit very similar peak metallicities, the MDF generated using Equation (2) displays a more prominent peak. This is the representative of the fact that Equation (2) accounts for the variation of period with amplitude as well as metallicity resulting in a cleaner abundance signature in the MDF. In the lower panel of Figure 12, we see that the peaks of the RR Lyrae MDFs in our two fields differ by $\sim 0.1$ dex; we find $\langle[\mathrm{Fe} / \mathrm{H}]\rangle=$ $-1.46 \pm 0.03$ for Field 1 and $\langle[\mathrm{Fe} / \mathrm{H}]\rangle=-1.54 \pm 0.03$ for Field 2, where the errors represent standard errors of the mean. This difference is not statistically significant.

Combining the RR Lyraes in the two fields yields the MDF shown as the solid line in Figure 13, wherein the binned and generalized histograms are shown. The latter has been constructed using an error of 0.31 dex per star (Alcock et al. 2000). The peak metallicity for our sample of ab-type RR Lyraes is then $\langle[\mathrm{Fe} / \mathrm{H}]\rangle=-1.50 \pm 0.02$, where the error is the standard error of the mean. The systematic error of this measurement is likely to be closer to $\sim 0.3$ dex. The dotted distributions in Figure 13 are the binned and generalized histograms for the RRab stars in the sample of B2004 scaled to the same number of ab-type RR Lyraes as in our fields. The peak abundance of this $\mathrm{MDF}$ is $\langle[\mathrm{Fe} / \mathrm{H}]\rangle=-1.77 \pm 0.06$.

There are three observations we can make regarding the appearance of Figure 13. First, it would seem that the errors on the individual metallicity measurements are significant enough to overwhelm any fine structure that may be present in the two MDFs. That is to say, both MDFs look essentially like normal distributions. Second, since we have applied the same transformation from period to metallicity to both sets of RR Lyraes, we can assert with significant statistical certainty that 


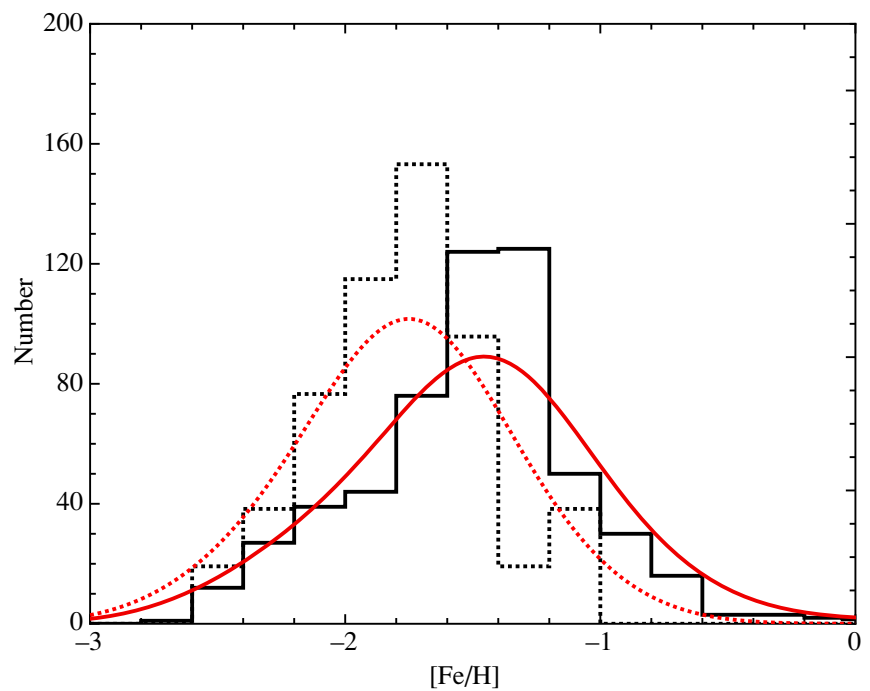

Figure 13. Comparison of the metallicity distribution function derived from the ab-type RR Lyraes from the present study (solid lines) and those from the Brown et al. (2004) study (dotted lines). The latter has been scaled to match the number of ab-type RR Lyraes in our two fields. Both binned and generalized histograms are shown.

the mean metal abundance of the RR Lyraes in the B2004 field is lower than that of the RR Lyraes in our two fields. This difference amounts to $\Delta[\mathrm{Fe} / \mathrm{H}]=0.27 \pm 0.06$ and reflects back on the period shift seen in the Bailey diagram shown in Figure 10. Third, there are a small but non-negligible number of ab-type RR Lyraes with metallicities above $[\mathrm{Fe} / \mathrm{H}] \sim-1$ that are not seen in the B2004 field. Given the fact that our field is closer to the central regions of M31 as compared with the B2004, it is possible that these metal-rich RR Lyraes could belong to the bulge or disk of M31. We return to this point in the following section.

One last point needs to be addressed before leaving this section and that is concerned with the M31 distance implied by the RR Lyraes in our sample. Using the relation advocated by Chaboyer $(1999)$ of $M_{V}(R R)=0.23[\mathrm{Fe} / \mathrm{H}]+0.93$ and a reddening of $E(B-V)=0.08 \pm 0.03$ (Schlegel et al. 1998), we find a distance modulus of $(m-M)_{0}=24.46 \pm 0.11$. This is consistent with the B2004 value and a number of previous determinations.

\section{DISCUSSION}

We now seek to place our RR Lyrae abundance results within the broader context of the projected radial metallicity distribution of various populations in the environs of M31. Figure 14 shows this information for a range of stellar populations in and around M31. The metallicities for the RR Lyraes in the two fields considered herein are shown by the filled circles, while the open circle represents the RR Lyraes in the B2004 field. The inner-most point is the bulge metallicity from the work of Sarajedini \& Jablonka (2005), while the remaining open squares are the bulge/halo points from the work of Kalirai et al. (2006) as shown in their Table 3 . The dashed line is the least-squares fit to the open squares with a slope of $-0.75 \pm 011$. The other points represent the dwarf spheroidal companions to M31 (crosses, Grebel et al. 2003; Koch \& Grebel 2006), the globular cluster G1 (filled square, Meylan et al. 2001), and the furthest globular cluster in M31 (open triangle, Martin et al. 2006). Note that we have adopted the mean metallicity of M32 from the work of Grillmair et al. (1996). The elongated rectangle represents the locations of the halo globular clusters in M33 from Sarajedini

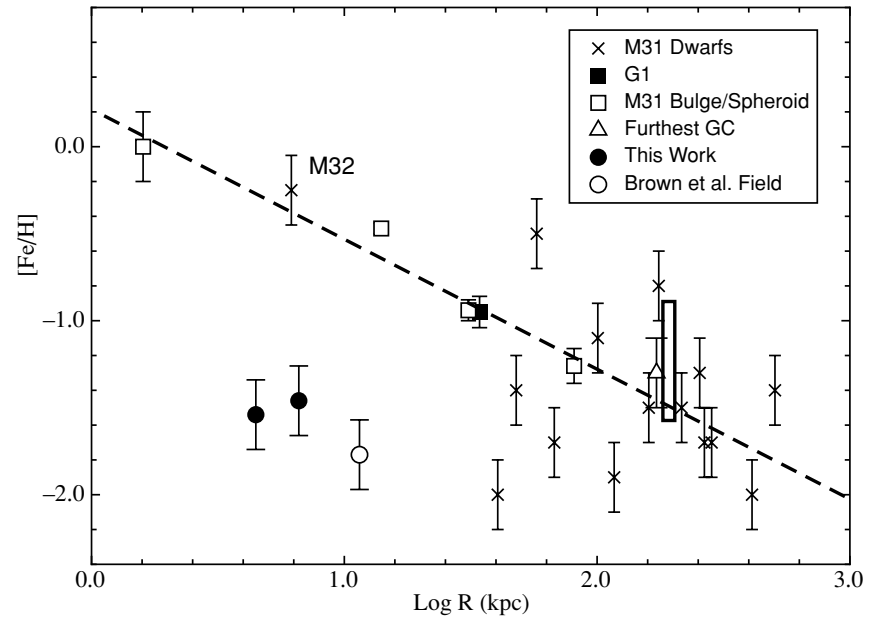

Figure 14. Plot of the variation of metal abundance with a projected distance from the center of M31. Our results are shown by the filled circles, while the mean metallicity of the RR Lyraes studied by Brown et al. (2004) is indicated by the open circle. The inner most open square represents the bulge abundance measured by Sarajedini \& Jablonka (2005). The remaining open squares are the bulge/halo points from the work of Kalirai et al. (2006). The dashed line is the least-squares fit to these data with a slope of $-0.75 \pm 0.11$. The crosses represent the dwarf galaxies surrounding M31 from the work of Grebel et al. (2003) and Koch \& Grebel (2006), whereas the abundance of M32 is taken from Grillmair et al. (1996). The filled square is the well known massive globular cluster G1 studied by Meylan et al. (2001). The open triangle is the furthest known globular cluster in M31 discovered by Martin et al. (2006). For completeness, the boxed region shows the location of the halo globular clusters in M33 from the work of Sarajedini et al. (2000). All of these points have been scaled to an M31 distance of $(m-M)_{0}=24.43$.

et al. (2000). All of these values are based on a distance of $(m-M)_{o}=24.43(770 \mathrm{kpc})$ for M31. In cases where an error in the metallicity is not available, we have adopted a value of \pm 0.2 dex.

We see in Figure 14 a clear representation of the notion that the halo population in M31 does not begin to dominate until a galactocentric distance of $\sim 30 \mathrm{kpc}$, as suggested by a number of authors (Guhathakurta et al. 2005; Irwin et al. 2005; Kalirai et al. 2006; Koch et al. 2008). At this location, we see a transition region between the globular cluster G1 which is consistent with the inner-spheroid metallicity gradient (dashed line) and the dwarf spheroidal galaxies which show no relation between abundance and galactocentric distance. In this sense, it would appear that the RR Lyrae populations in our field and that of B2004 follow the trend outlined by the stellar populations outside of $\sim 30 \mathrm{kpc}$. This suggests that the RR Lyraes at these locations are probably members of the M31 halo rather than its bulge suggesting that the halo can be studied as close as $4 \mathrm{kpc}$ from the center of M31 by focusing on the RR Lyraes.

\section{SUMMARY AND CONCLUSIONS}

We have presented F606W $(\sim V)$ observations from the $H S T$ archive taken with the Advanced Camera for Surveys of two fields located 4-6 kpc from the center of M31. In these regions, we identify 752 variable stars of which 681 are likely to be bona fide RR Lyraes. From the properties of these stars, we draw the following conclusions.

1. The mean magnitude of the RR Lyrae stars is $\langle V\rangle=$ $25.29 \pm 0.05$ where the uncertainty combines both the random and systematic errors. This is in good agreement with the results of Brown et al. (2004).

2. The ratio of c-type RR Lyraes to all types is reminiscent of the RR Lyraes in Oosterhoff type I globular clusters in 
the Milky Way. This ratio is significantly different than the Brown et al. (2004) field at $11 \mathrm{kpc}$ from the center of M31 wherein this ratio is closer to that of Oosterhoff II clusters.

3. When the periods and amplitudes of the ab-type RR Lyraes in our sample are interpreted in terms of metallicity, we find the metallicity distribution function to be indistinguishable from a Gaussian with a peak at $\langle[\mathrm{Fe} / \mathrm{H}]\rangle=-1.50 \pm 0.02$, where the error is the standard error of the mean. The same analysis applied to the Brown et al. (2004) RR Lyraes yields a peak of $\langle[\mathrm{Fe} / \mathrm{H}]\rangle=-1.77 \pm 0.06$.

4. Using the RR Lyrae luminosity-metallicity relation advocated by Chaboyer (1999) and a reddening of $E(B-V)=$ $0.08 \pm 0.03$, we find a distance modulus of $(m-M)_{0}=$ $24.46 \pm 0.11$ for M31.

5. We examine the radial metallicity gradient in the environs of M31 using published values for the bulge and halo of M31 as well as the abundances of the dwarf spheroidal companions and globular clusters of M31. In this context, despite the relative proximity of the RR Lyraes in the present study to the center of M31, their metal abundance is more reminiscent of a halo population than a bulge or disk. Therefore, by using the RR Lyraes as a proxy, the halo can be studied as close as $4 \mathrm{kpc}$ from the center of M31.

We are grateful to Andy Layden, Nathan De Lee, and Karen Kinemuchi for useful conversations as this manuscript was being written. A.S. is grateful for support from NASA through grant AR-11277.01-A from the Space Telescope Science Institute, which is operated by the Association of Universities for Research in Astronomy, Inc., for NASA under contract NAS526555. The National Optical Astronomy Observatory is operated by AURA, Inc., under cooperative agreement with the National Science Foundation.

\section{REFERENCES}

Alcock, C., et al. 2000, AJ, 119, 2194

Alonso-García, J., Mateo, M., \& Worthey, G. 2004, AJ, 127, 868
Brown, T. M., et al. 2004, AJ, 127, 2738

Castellani, M., Caputo, F., \& Castellani, V. 2003, A\&A, 410, 871

Chaboyer, B. 1999, in Astrophysics and Space Science Library 237, PostHipparcos cosmic candles, ed. A. Heck \& F. Caputo (Dordrecht: Boston), 111

Clement, C. M. 2000, in ASP Conf. Ser. 203, Impact of Large Scale Surveys on Pulsating Star Research IAU Colloq.176, ed. L. Szabados \& D. W. Kurtz (San Francisco, CA: ASP), 266

Clementini, G., Federici, L., Corsi, C., Cacciari, C., Bellazzini, M., \& Smith, H. A. 2001, ApJ, 559, L109

Contreras, R., et al. 2008, Mem. Soc. Astron. Ital., 79, 686

Dolphin, A. E., Saha, A., Olzsewski, E., Thim, F., Skillman, E. D., Gallagher, J. J., \& Hoessel, J. 2004, AJ, 127, 875

Durrell, P. R., Harris, W. E., \& Pritchet, C. J. 2001, AJ, 121, 2557

Freedman, W. L., \& Madore, B. F. 1990, ApJ, 365, 186

Grebel, E. K., Gallagher, J. S., III, \& Harbeck, D. 2003, AJ, 125, 1926

Grillmair, C., et al. 1996, AJ, 112, 1975

Guhathakurta, P., et al. 2005, arXiv:astro-ph/0502366

Horne, J. H., \& Baliunas, S. L. 1986, ApJ, 302, 757

Irwin, M. J., Ferguson, A. M. N., Ibata, R. A., Lewis, G. F., \& Tanvir, N. R. 2005, ApJ, 628, L108

Kalirai, J. S., et al. 2006, ApJ, 648, 389

Koch, A., \& Grebel, E. K. 2006, AJ, 131, 1405

Koch, A., et al. 2008, ApJ, 689, 958

Layden, A. C. 1995, AJ, 110, 2312

Layden, A. C., \& Sarajedini, A. 2000, AJ, 119, 1760

Mack, J., Gilliland, R. L., Anderson, J., \& Sirianni, M. 2007, ACS Instrum. Sci. Rep. 2007-02

Mahmud, N., \& Anderson, J. 2008, PASP, 120, 907

Martin, N. F., et al. 2006, MNRAS, 371, 1983

Meylan, G., Sarajedini, A., Jablonka, P., Djorgovski, S. G., Bridges, T., \& Rich, R. M. 2001, AJ, 122, 830

Mould, J., \& Kristian, J. 1986, ApJ, 305, 591

Pritchet, C. J., \& van den Bergh, S. 1987, ApJ, 316, 517

Reiss, A., \& Mack, J. 2004, ACS Instrum. Sci. Rep. 2004-06

Sandage, A. R. 1993, AJ, 106, 687

Sarajedini, A., Barker, M., Geisler, D., Harding, P., \& Schommer, R. 2006, AJ, 132,1361

Sarajedini, A., \& Jablonka, P. 2005, AJ, 130, 1627

Sarajedini, A., et al. 2000, AJ, 120, 2437

Scargle, J. D. 1982, ApJ, 263, 835

Schlegel, D. J., Finkbeiner, D. P., \& Davis, M. 1998, ApJ, 500, 525

Sirianni, M., et al. 2005, PASP, 117, 1049

Stetson, P. B. 1987, PASP, 99, 191

Stetson, P. B. 1994, PASP, 106, 250 